\title{
Alterations in cardiac adrenergic signaling and calcium cycling differentially affect the progression of cardiomyopathy
}

\author{
Kalev Freeman, ${ }^{1}$ Imanuel Lerman, ${ }^{1}$ Evangelia G. Kranias, ${ }^{2}$ Teresa Bohlmeyer, ${ }^{3}$ \\ Michael R. Bristow, ${ }^{3}$ Robert J. Lefkowitz, ${ }^{4}$ Guido Iaccarino, ${ }^{5}$ Walter J. Koch, ${ }^{5}$ \\ and Leslie A. Leinwand ${ }^{1}$ \\ ${ }^{1}$ Department of Molecular, Cellular, and Developmental Biology, University of Colorado, Boulder, Colorado, USA \\ ${ }^{2}$ Department of Pharmacology and Cell Biophysics, University of Cincinnati College of Medicine, Cincinnati, Ohio, USA \\ ${ }^{3}$ Division of Cardiology, University of Colorado Health Sciences Center, Denver, Colorado, USA \\ ${ }^{4}$ Department of Medicine, and \\ ${ }^{5}$ Department of Surgery, Duke University Medical Center, Durham, North Carolina, USA
}

Address correspondence to: Leslie A. Leinwand, Department of Molecular, Cellular, and Developmental Biology, University of Colorado, Campus Box 347, Boulder, Colorado 80309-0347, USA.

Phone: (303) 492-7606; Fax: (303) 492-8907; E-mail: Leslie.Leinwand@Colorado.Edu.

Received for publication December 29, 2000, and accepted in revised form February 26, 2001.

The medical treatment of chronic heart failure has undergone a dramatic transition in the past decade. Short-term approaches for altering hemodynamics have given way to long-term, reparative strategies, including $\beta$-adrenergic receptor ( $\beta \mathrm{AR}$ ) blockade. This was once viewed as counterintuitive, because acute administration causes myocardial depression. Cardiac myocytes from failing hearts show changes in $\beta A R$ signaling and excitation-contraction coupling that can impair cardiac contractility, but the role of these abnormalities in the progression of heart failure is controversial. We therefore tested the impact of different manipulations that increase contractility on the progression of cardiac dysfunction in a mouse model of hypertrophic cardiomyopathy. High-level overexpression of the $\beta_{2}$ AR caused rapidly progressive cardiac failure in this model. In contrast, phospholamban ablation prevented systolic dysfunction and exercise intolerance, but not hypertrophy, in hypertrophic cardiomyopathy mice. Cardiac expression of a peptide inhibitor of the $\beta A R$ kinase 1 not only prevented systolic dysfunction and exercise intolerance but also decreased cardiac remodeling and hypertrophic gene expression. These three manipulations of cardiac contractility had distinct effects on disease progression, suggesting that selective modulation of particular aspects of $\beta A R$ signaling or excitation-contraction coupling can provide therapeutic benefit.

J.Clin. Invest. 107:967-974 (2001).

\section{Introduction}

Chronic heart failure, a leading cause of morbidity and mortality, can result from a variety of primary insults to the heart, including genetic cardiomyopathies. Early in the course of myocardial failure, the heart compensates for inadequate function by adrenergic stimulation, activation of the renin-angiotensin-aldosterone system and other neurohumoral/cytokine systems, and myocardial hypertrophy. However, over time these compensatory mechanisms cease to provide benefit, and a pathological decompensation often ensues in which cardiac function deteriorates, the ventricles become dilated and fibrotic, and ultimately the heart fails. The molecular bases of the deterioration in the heart's pumping capacity and the associated pathological remodeling process are topics of intense investigation.

$\beta$-adrenergic receptor ( $\beta$ AR) blockade has been shown to provide therapeutic benefit in chronic heart failure (1-3). It has been hypothesized that inhibiting $\beta A R$ signal transduction adds to the failing heart's endogenous antiadrenergic strategy, which includes receptor downregulation and uncoupling, thereby minimizing adrenergic toxicity (4). Nonetheless, the viewpoint that $\beta A R$ signaling is toxic to the failing heart may be an overly broad generalization. The toxic effects of elevated adrenergic drive are mediated primarily by $\beta_{1}$ ARs, in contrast to $\beta_{2}$ AR stimulation, which may be protective in some settings (5). Furthermore, manipulations that increase cardiac contractility, either via enhanced $\beta A R$ signaling or alteration of the phospholamban-SR $\mathrm{Ca}^{2+}$ ATPase interaction, have recently been shown to prevent heart failure in several cardiomyopathic mouse models (6-9). Determining whether manipulation of regulatory proteins involved in $\mathrm{Ca}^{2+}$ handling or cellular signaling is helpful or harmful in heart disease requires further investigation (10).

We therefore tested the therapeutic impact of three different genetic manipulations that increase contractility on the progression of cardiac dysfunction in a mouse model of hypertrophic cardiomyopathy (HCM). 

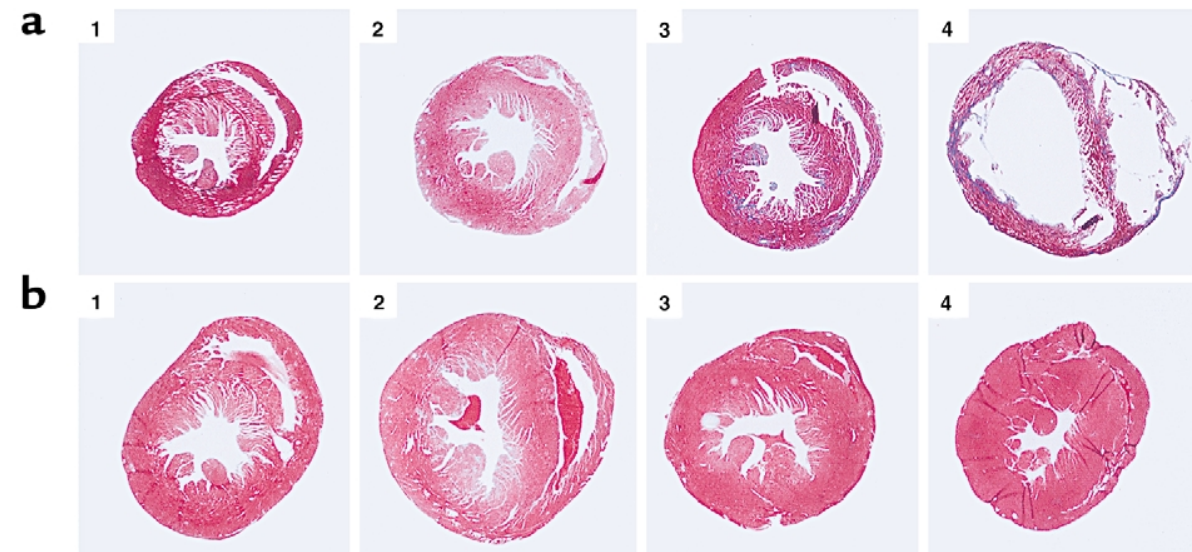

C 1
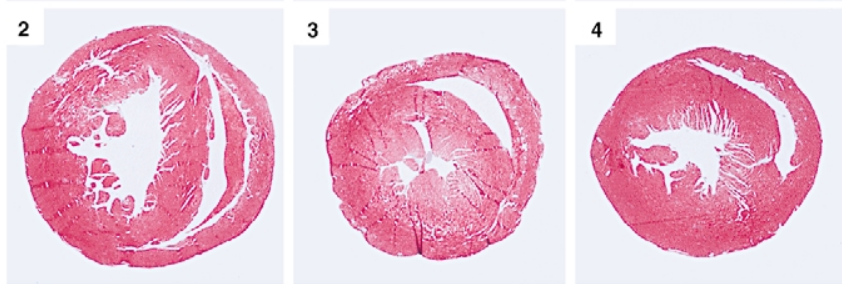

\section{Figure 1}

Histology of cross-bred mouse hearts. (a) Representative cross-sections of the hearts from $\mathrm{HCM} \times \beta_{2} \mathrm{AR}$ offspring: panel 1, NTG; panel 2, HCM; panel 3, $\beta_{2} \mathrm{AR}$; panel 4, HCM $/ \beta_{2} \mathrm{AR}$. (b) HCM $\times \beta$ ARKct offspring: panel 1, NTG; panel 2, HCM; panel 3, $\beta$ ARKct; panel 4, $\mathrm{HCM} / \beta$ ARKct. (c) HCM $\times$ PLB-null offspring: panel 1, NTG; panel 2, HCM; panel 3, PLB-null; panel 4, HCM/PLB-null. Magnification, $\times 5$.

In this model, male mice begin to show signs of heart failure by 8 months of age, with a transition from cardiac hypertrophy to ventricular dilation accompanied by exercise intolerance, cardiac dysfunction, and signs of adrenergic desensitization $(11,12) . \beta_{2}$ AR overexpression, $\beta$ AR kinase inhibition, and phospholamban ablation each had distinct effects on disease progression, suggesting that selective modulation of particular aspects of $\beta A R$ signaling or cardiac excitation-contraction coupling can provide therapeutic benefit.

\section{Methods}

Experimental animals. HCM mice heterozygous for a mutant myosin heavy chain transgene (R403Q missense mutation with an additional deletion of amino acids 468-527 bridged by nine nonmyosin amino acids) (11) were crossed with heterozygous animals from the TG4 line that overexpress the $\beta_{2}$ adrenergic receptor $\left(\beta_{2} \mathrm{AR}\right.$ mice) (13), to generate double-heterozygous $\mathrm{HCM} / \beta_{2} \mathrm{AR}$ transgenic animals, along with $\mathrm{HCM}, \beta_{2} \mathrm{AR}$, and nontransgenic (NTG) littermate controls. Similarly, HCM heterozygotes were crossbred with mice expressing the $\mathrm{COOH}$-terminus of the $\beta$ adrenergic receptor kinase 1 ( $\beta$ ARKct mice), which inhibits activation of endogenous $\beta$ ARK1 (14), to yield HCM/ $\beta$ ARKct double transgenic animals and appropriate littermate controls. HCM heterozygotes were also crossbred with phospholamban-null (PLB-null) animals (15). The resulting offspring possessed one PLB-null allele; animals from this group that were also heterozygous for the HCM transgene were crossed with offspring that were negative for the HCM transgene to obtain animals heterozygous for the HCM transgene that were also PLB-null (HCM/PLB-null mice) and littermate controls. The background strain of all mice used was C57BL/6J, except PLB-null, which were in a FVB/CF1 mixed background, so age-matched littermate controls were used in all experiments to eliminate potential strain differences; furthermore, only male animals were used for these studies. The first study group from each cross underwent serial echocardiography at 4,8 , and 12 months. Separate groups of animals underwent exercise studies followed by sacrifice for histology at 8 months of age, or were sacrificed at 8 months of age for RNA analysis. Animals were handled according to approved protocols of the University of Colorado.

Echocardiography. Serial echocardiography was performed on animals at 4-, 8-, and 12-month time points at near-physiological heart rates $(560 \pm 15$ beats per minute) as described previously (12). A Vingmed System Five echocardiography machine (GE Medical Systems, Milwaukee, Wisconsin, USA) with a 10$\mathrm{MHz}$-phased array transducer was used for image acquisition, and digital measurements of left-ventricular percent fractional shortening (\%FS) were made from $M$-mode images that were regenerated from the original R $\theta$ sampling information.

Treadmill exercise tolerance. Mice were exercised on a custom-built eight-lane treadmill with an infrared detection system above the shock stimulus, as described previously (12). The animals were acclimated to the treadmill over a 1-week period, which consisted 
of one 15 -minute low-speed $(5-7 \mathrm{~m} / \mathrm{min})$ session without the shock grid, and two 15 -minute sessions with the shock-grid (5-7 and $20 \mathrm{~m} / \mathrm{min}$, respectively). After the acclimation period, the animals were exercised six times each at $20 \mathrm{~m} / \mathrm{min}$ on a 7 degree incline. If an animal became exhausted during the experiment, the shock bars for that animal were turned off and the animal was removed from the apparatus. The performance for each mouse was assessed as the average number of beam-breaks per minute over all exercise sessions.

Histology. Hearts were rapidly excised following cervical dislocation and placed in PBS while still beating to allow blood to be pumped out of the cardiac chambers and coronary vessels. Hearts were then placed in a 10:1 volume of $10 \%$ neutral buffered formalin to tissue for fixation. The fixed hearts were processed, embedded in paraffin, sectioned, and stained with Masson's Trichrome according to standard protocols.

$R N A$ analysis. Mice were sacrificed by cervical dislocation, and hearts were placed in PBS to allow the extrusion of blood as just described. Left ventricles were then dissected and frozen at $-80^{\circ} \mathrm{C}$ within 10 minutes of sacrifice, and total RNA was extracted using TRIzol Reagent (Life Technologies Inc., Grand Island, New York, USA). mRNA levels of $\beta$ myosin heavy chain $(\beta \mathrm{MyHC}), \alpha$ skeletal actin (sACT), and atrial natriuretic factor (ANF) were assessed by slot-blot using oligonucleotide probes described previously $(16,17)$. Blots were exposed on phosphor storage screens and scanned using a phosphoimager, and the signal intensities were digitally quantified. Small variations in loading were corrected by normalization to GAPDH mRNA levels, which were determined using duplicate blots as described previously (12).

Statistics. Data are presented as mean \pm SEM. The number of mice used in each experiment is indicated. Statistical analysis was performed by ANOVA for multiple group comparisons.

\section{Results}

To evaluate the impact of $\beta_{2}$ AR overexpression, expression of the $\beta$ ARKct peptide that can inhibit $\beta$ ARK1, and ablation of phospholamban on the progression of cardiomyopathy, animals with these manipulations (13-15) were cross-bred into the HCM mouse line, and male offspring were studied through 1 year of age. In the offspring of the $\mathrm{HCM} \times \beta_{2} \mathrm{AR}$ cross, we first noted that the $\mathrm{HCM} / \beta_{2} \mathrm{AR}$ double transgenic animals had significantly increased mortality, with only $50 \%$ survival at 8 months and less than $10 \%$ survival at 12 months. Some animals showed physical signs of congestive heart failure, including extensive intraperitoneal edema, pulmonary and hepatic enlargement, and massively enlarged hearts (as great as $430 \mathrm{mg}$ in a

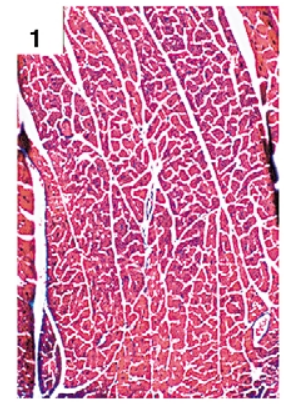

b

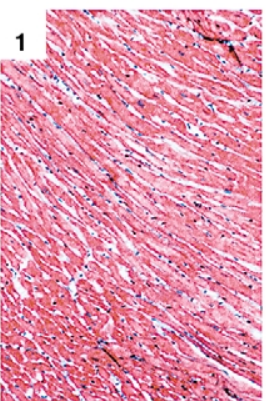

C

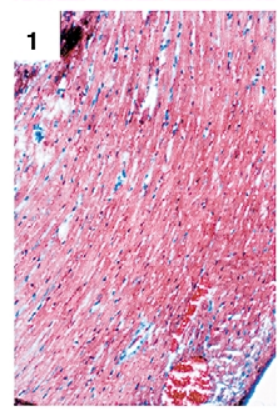

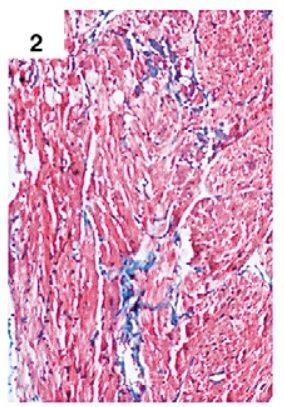
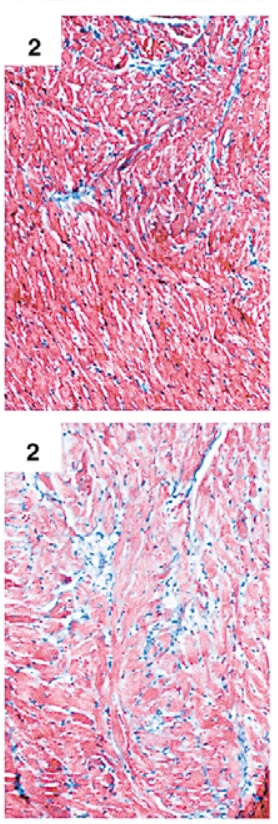
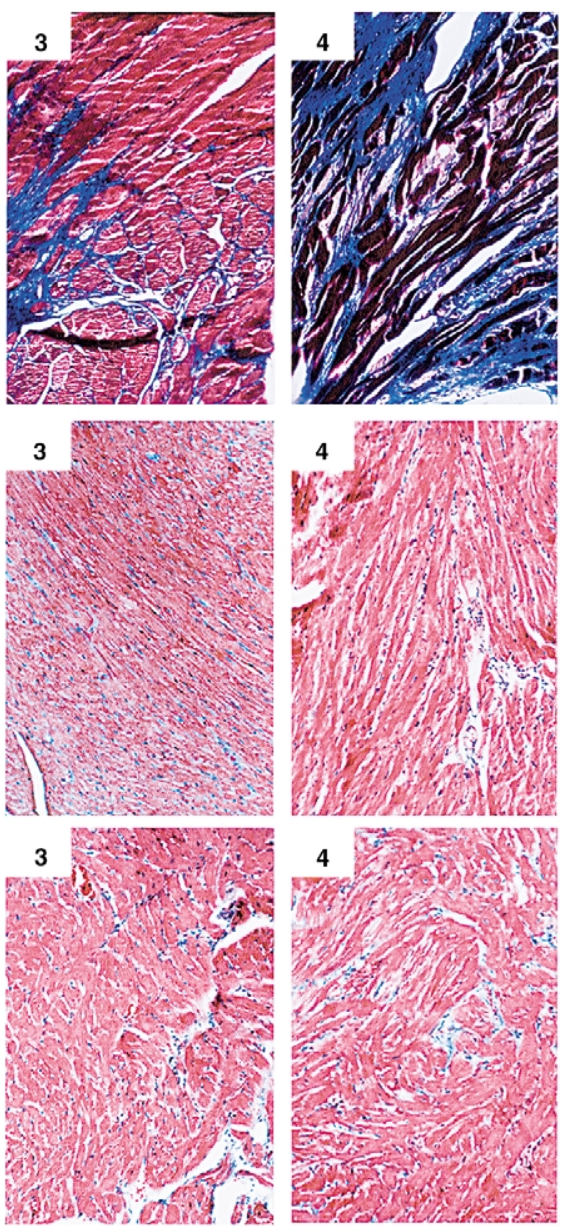
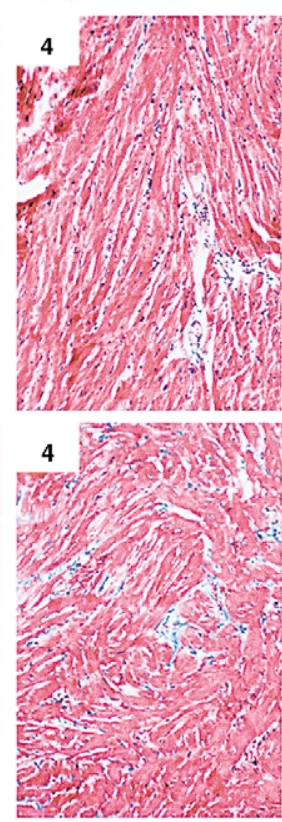

\section{Figure 2}

Histology of cross-bred mouse hearts. (a) $\mathrm{HCM} \times \beta_{2} \mathrm{AR}$ offspring: panel 1, NTG; panel 2, HCM; panel 3, $\beta_{2} A R$; panel 4, $\mathrm{HCM} / \beta_{2} \mathrm{AR}$. (b) $\mathrm{HCM} \times$ $\beta A R K c t$ offspring: panel 1, NTG; panel 2, HCM; panel 3, $\beta A R K c t$; panel 4, HCM/ $\beta$ ARKct. (c) HCM $\times$ PLB-null offspring: panel 1, NTG; panel 2, HCM; panel 3, PLB-null; panel 4, HCM/PLB-null. Magnification $\times 250$. 
one 13-week-old mouse) with atrial and ventricular dilation. Both the HCM and $\beta_{2} \mathrm{AR}$ littermates also showed increased mortality compared with NTG controls at one year of age. No differences in survival were noted in any of the offspring from $\mathrm{HCM} \times \beta A R K c t$ or $\mathrm{HCM} \times$ PLB-null crosses.

Compared with NTG controls, HCM mice showed modest cardiac hypertrophy with an increased left ventricular internal area, as described previously (Figure 1, first two columns) $(11,12)$. The gross morphology of hearts from the $\beta_{2} \mathrm{AR}, \beta \mathrm{ARKct}$, and PLB-null animals was not different from that of NTG control hearts (Figure 1 , third column). The morphology of $\mathrm{HCM} / \beta A R K c t$ and HCM/PLB-null hearts was also normal, but the $\mathrm{HCM} / \beta_{2} \mathrm{AR}$ hearts showed dilated ventricular and atrial chambers with thin, fibrotic ventricular walls (Figure 1, fourth column).

The hearts of HCM mice from the $\mathrm{HCM} \times \beta A R K c t$ and $\mathrm{HCM} \times$ PLB-null crosses were significantly hypertrophic (respectively, $17 \%$ and $10 \%$ greater than those of NTG controls); the HCM mice from the HCM $\times$ $\beta_{2}$ AR cross also showed a trend to cardiac hypertrophy that was not statistically significant in multigroup

a

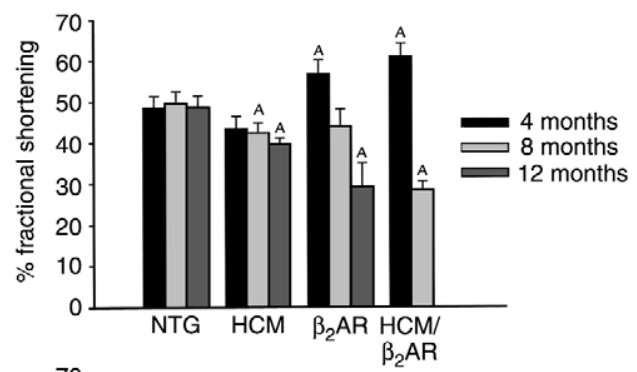

b

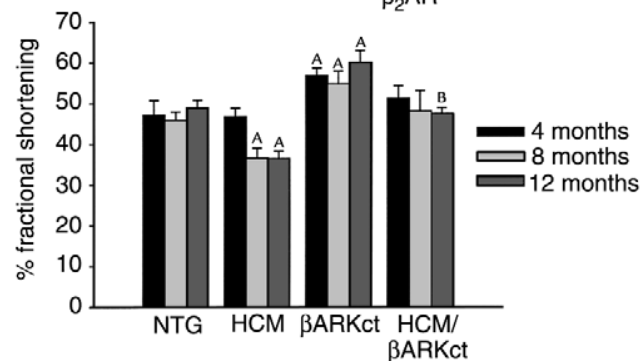

c

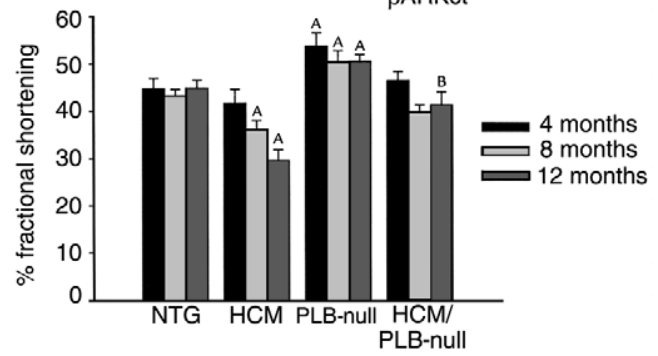

Figure 3

Systolic function of cross-bred mouse hearts as determined by serial echocardiography. (a) $\mathrm{HCM} \times \beta_{2} \mathrm{AR}$ mice (NTG, $n=8$; $\mathrm{HCM}, n=12$; $\left.\beta_{2} \mathrm{AR}, n=7 ; \mathrm{HCM} / \beta_{2} \mathrm{AR}, n=4\right)$. (b) $\mathrm{HCM} \times \beta \mathrm{ARKct}$ mice (NTG, $n=$ 5 ; HCM, $n=6$; $\beta$ ARKct, $n=8$; HCM $/ \beta A R K c t, n=5)$. (c) HCM $\times$ PLBnull mice (NTG, $n=6$; HCM, $n=8$; PLB-null, $n=7$; HCM/PLB-null, $n=9) .{ }^{A} P<0.05$ vs. NTG; ANOVA. ${ }^{B} P<0.05$ vs. HCM; ANOVA. comparisons that included the more extreme hypertrophy of $\mathrm{HCM} / \beta_{2} \mathrm{AR}$ littermates (Table 1$)$. $\beta_{2} \mathrm{AR}$ hearts were not hypertrophied, but the heart/body weight ratios of $\mathrm{HCM} / \beta_{2} \mathrm{AR}$ littermates were approximately $67 \%$ greater than those of NTG controls. Cardiac hypertrophy was not apparent in $\beta A R K c t$ or HCM/ $\beta A R K c t$ animals. In contrast, PLB-null mice showed a modest but significant increase in heart/body weight ( $10 \%$ greater than that of NTG controls). The heart/body weights of HCM/PLB-null mice were nearly $30 \%$ greater than those of NTG controls and were also significantly greater than those of either HCM or PLB-null littermates.

The characteristic histopathology of hypertrophic cardiomyopathy, including fibrosis and myocellular disarray, was apparent to a variable extent in all mice that carried the mutant myosin transgene, including the $\mathrm{HCM} / \beta_{2} \mathrm{AR}, \mathrm{HCM} / \beta \mathrm{ARKct}$, and HCM/PLB-null mice, compared with NTG controls (Figure 2). The hearts of 8-month-old $\beta_{2} \mathrm{AR}$ mice showed areas of significant fibrosis, but without myocellular disarray (Figure $2 \mathrm{a}$, third column). $\mathrm{HCM} / \beta_{2} \mathrm{AR}$ hearts showed severe histopathology, with extensive fibrosis and disarray (Figure 2a, fourth column). The $\beta A R K c t$ and PLBnull mice showed normal myocardial histology (Figure $2, \mathrm{~b}$ and $\mathrm{c}$, third column).

We performed transthoracic echocardiography on agematched male littermates at 4, 8, and 12 months of age and measured \%FS of the left ventricle for each mouse. The HCM mice had normal \%FS at 4 months of age, with decreased \%FS at both 8 and 12 months of age (Figure 3a). We noted a significant increase in systolic function in both the $\beta_{2} \mathrm{AR}$ littermates and the $\mathrm{HCM} / \beta_{2} \mathrm{AR}$ double transgenics at 4 months of age. In the $\beta_{2} A R$ animals, the increased \%FS was no longer observed at 8 months of age, and by 12 months, systolic function was significantly impaired. The $\mathrm{HCM} / \beta_{2} \mathrm{AR}$ double transgenics showed an even more dramatic loss of cardiac function, with a \%FS of only two-thirds that of littermate controls at 8 months of age. Only one $\mathrm{HCM} / \beta_{2} \mathrm{AR}$ animal from this study group survived to 12 months of age, and he died during preparation for echocardiography.

The $\beta A R K c t$ mice displayed a marked increase in cardiac function at 4 months of age that persisted through one year (Figure $3 \mathrm{~b}$ ). Unlike the $\mathrm{HCM} / \beta_{2} \mathrm{AR}$ mice, which were hypercontractile at 4 months of age, the HCM/ $\beta A R K c t$ did not have higher contractility than NTG controls did at any time point. However, expression of the $\beta A R K c t$ transgene prevented the depression in cardiac dysfunction that occurred in HCM littermates, and at 1 year of age, \%FS was significantly higher in the HCM/ $\beta$ ARKct mice than in their HCM littermates. HCM $\times$ PLB-null offspring showed a pattern of systolic function similar to that of HCM $\times \beta A$ ARKct offspring (Figure 3c). Systolic function was greatly enhanced in PLB-null mice at 4, 8, and 12 months of age. The \%FS of HCM/PLB-null mice was neither increased nor decreased relative to controls through 1 year, and \%FS was significantly increased compared 
Table 1

Heart and body weights of cross-bred mice

\begin{tabular}{|c|c|c|c|c|c|}
\hline Cross & Genotype $(n)$ & Age (mo) & Body weight (g) & Heart weight (mg) & Heart wt/Body wt \\
\hline \multirow[t]{4}{*}{$\mathrm{HCM} \times \beta_{2} \mathrm{AR}$} & NTG (9) & $9.71 \pm 0.09$ & $30.26 \pm 0.80$ & $146.2 \pm 3.2$ & $4.87 \pm 0.21$ \\
\hline & $\operatorname{HCM}(8)$ & $9.65 \pm 0.19$ & $30.23 \pm 1.50$ & $165.3 \pm 7.7$ & $5.49 \pm 0.18$ \\
\hline & $\beta_{2} \operatorname{AR}(8)$ & $9.43 \pm 0.21$ & $30.25 \pm 0.53$ & $141.1 \pm 4.3$ & $4.99 \pm 0.10$ \\
\hline & $\mathrm{HCM} / \beta_{2} \mathrm{AR}(7)$ & $9.90 \pm 0.11$ & $32.11 \pm 0.76$ & $265.3 \pm 39.8^{\mathrm{B}}$ & $8.18 \pm 1.06^{\mathrm{B}}$ \\
\hline \multirow[t]{4}{*}{ HCM $\times \beta A R K c t$} & NTG (13) & $10.93 \pm 0.36$ & $32.46 \pm 0.59$ & $150.3 \pm 3.6$ & $4.63 \pm 0.08$ \\
\hline & $\operatorname{HCM}(12)$ & $10.55 \pm 0.25$ & $31.20 \pm 0.66$ & $169.5 \pm 14.2^{\mathrm{A}}$ & $5.45 \pm 0.45^{\mathrm{A}}$ \\
\hline & $\beta A R K c t$ (15) & $10.50 \pm 0.25$ & $32.70 \pm 0.57$ & $145.0 \pm 3.5$ & $4.45 \pm 0.12$ \\
\hline & HCM/ßARKct (13) & $10.02 \pm 0.48$ & $31.46 \pm 0.89$ & $150.5 \pm 3.9$ & $4.80 \pm 0.10$ \\
\hline \multirow[t]{4}{*}{ HCM $\times$ PLB-null } & NTG (15) & $10.51 \pm 0.05$ & $34.03 \pm 1.15$ & $167.2 \pm 7.1$ & $4.91 \pm 0.12$ \\
\hline & $\operatorname{HCM}(12)$ & $10.65 \pm 0.11$ & $35.43 \pm 2.04$ & $188.9 \pm 10.4^{\mathrm{A}}$ & $5.40 \pm 0.23^{\mathrm{A}}$ \\
\hline & PLB-null (15) & $10.52 \pm 0.07$ & $33.86 \pm 0.92$ & $183.5 \pm 8.0^{\mathrm{A}}$ & $5.41 \pm 0.16^{\mathrm{A}}$ \\
\hline & HCM/PLB-null (15) & $10.46 \pm 0.03$ & $32.93 \pm 0.96$ & $206.7 \pm 5.1^{\mathrm{B}, \mathrm{C}}$ & $6.33 \pm 0.19^{B, C}$ \\
\hline
\end{tabular}

${ }^{A} P<0.05$ vs. NTG; ANOVA. ${ }^{B} P<0.001$ vs. NTG; ANOVA. ${ }^{C} P<0.001$ vs. HCM and PLB-null; ANOVA.

with HCM littermates at 1 year of age. Like $\beta$ ARK inhibition, ablation of PLB appeared to prevent cardiac dysfunction in the older male HCM animals without causing a significant increase in inotropy over NTG controls at any time point.

We previously observed impaired exercise tolerance in HCM mice at 8 months of age (12), and we used a similar treadmill exercise test to evaluate $\mathrm{HCM} \times \beta_{2} \mathrm{AR}$, $\mathrm{HCM} \times \mathrm{BARKct}$, and $\mathrm{HCM} \times \mathrm{PLB}$-null offspring. The $\mathrm{HCM} \times \beta A R K c t$ and $\mathrm{HCM} \times$ PLB-null mice were studied at 8 months of age; owing to the higher mortality of $\mathrm{HCM} / \beta_{2} \mathrm{AR}$ mice, the $\mathrm{HCM} \times \beta_{2} \mathrm{AR}$ offspring were studied at 6 months of age. In the HCM $\times \beta_{2}$ AR experiment, we noted significantly decreased exercise tolerance in the HCM mice, seen as an increased number of beam breaks per minute (Figure 4a). The 6 -month-old $\beta_{2} \mathrm{AR}$ mice showed significantly increased exercise tolerance, with a decreased number of beam breaks per minute. Two of the $8 \mathrm{HCM} / \beta_{2} \mathrm{AR}$ animals in this group died before beginning exercise testing, and a third mouse died during the first exercise session. Exercise tolerance in the five surviving $\mathrm{HCM} / \beta_{2} \mathrm{AR}$ mice was slightly but not significantly impaired. In studies of $\mathrm{HCM} \times \beta$ ARKct and HCM $\times$ PLB-null animals, HCM mice showed impaired exercise tolerance (Figure 4, b and c). Exercise tolerance of the PLB-null animals was not different from that of NTG, as reported previously (18); $\beta$ ARKct mice also exhibited normal exercise tolerance. Both the $\mathrm{HCM} / \beta A R K c t$ double transgenic mice and HCM/PLBnull mice showed significantly improved exercise tolerance compared with HCM littermates.

$\beta \mathrm{MyHC}, \mathrm{ANF}$, and sACT are normally expressed in the developing mouse heart, and induction of these genes has been well-established in cardiac hypertrophy $(19,20)$. We previously observed an upregulation of $\beta \mathrm{MyHC}, \mathrm{ANF}$, and sACT mRNA in hearts of 8-monthold male HCM mice (12). We assessed mRNA levels of $\beta \mathrm{MyHC}, \mathrm{ANF}$, and sACT in the left ventricular myocardium of 8-month-old crossbred animals (Figure 5). Neither $\beta_{2} A R$ overexpression nor PLB ablation reduced the expression of hypertrophic markers in
HCM hearts. $\beta \mathrm{MyHC}$ mRNA was markedly elevated in both $\beta_{2} \mathrm{AR}$ mice and $\mathrm{HCM} / \beta_{2} \mathrm{AR}$ hearts $(\sim 25$ times that of NTG littermates, and 12 times that of HCM littermates, respectively Figure 5a). Neither ANF nor sACT was upregulated in the $\beta_{2} \mathrm{AR}$ mice, but expression of these genes was elevated in the $\mathrm{HCM}$ and $\mathrm{HCM} / \beta_{2} \mathrm{AR}$ mice. The three mRNA levels were also significantly elevated in HCM/PLB-null mice (Figure 5c). In contrast, the mRNA levels of both ANF and sACT, but not $\beta \mathrm{MyHC}$, were significantly less in HCM/ $\beta$ ARKct mice than in HCM littermates (Figure 5b).

\section{Discussion}

We found that high-level overexpression of the $\beta_{2} \mathrm{AR}$ resulted in enhanced cardiac function at early time points, but it caused toxic effects at later time points even in the absence of the HCM transgene. We found significantly improved exercise tolerance and increased $\% \mathrm{FS}$ in $\beta_{2} \mathrm{AR}$ mice between 4 and 6 months of age. To our knowledge, this is the first report of a transgenic manipulation that resulted in an increased ability to perform exercise, and it is probably a result of the markedly enhanced cardiac contractility in these animals. Similarly, in the HCM background, high-levels of $\beta_{2} \mathrm{AR}$ overexpression caused a significant increase in \%FS at 4 months of age, suggesting an initial benefit. The effects of this genetic manipulation may be analogous to the acute positive effect seen with $\beta A R$ agonists. However, the hypercontractility observed in younger $\beta_{2} \mathrm{AR}$ animals was no longer present at 8 months of age, and histology revealed myocardial fibrosis. By 1 year, we observed elevated mortality in the $\beta_{2} \mathrm{AR}$ mice, and surviving animals had significantly impaired systolic function. In the HCM background, $\beta_{2}$ AR overexpression ultimately resulted in severe heart failure, with increased mortality, extreme cardiac dilation and fibrosis, and impaired cardiac function.

Toxic effects of $\beta A R$ signaling on the heart have previously been observed in response to isoproterenol administration, extremely high levels of $\beta_{2}$ AR overexpression ( $\geq 100$-fold) $(8,21)$, low levels of $\beta_{1}$ AR overex- 
a

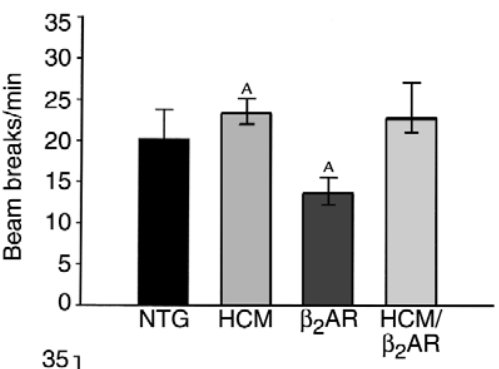

b

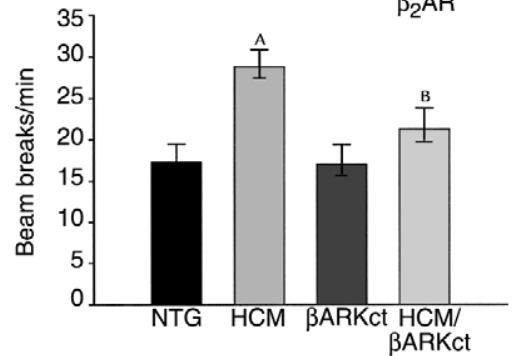

C

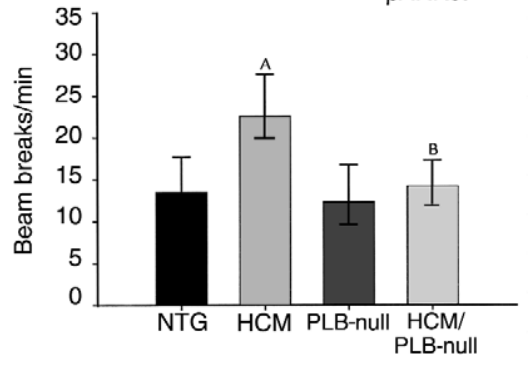

\section{Figure 4}

Treadmill exercise tolerance of cross-bred mice measured as beam breaks per minute. Mice were run five times for 1 hour at $20 \mathrm{~m} / \mathrm{min}$, and scores for each animal were averaged. (a) $\mathrm{HCM} \times \beta_{2} \mathrm{AR}$ mice (NTG, $n=8 ; \mathrm{HCM}, n=6 ; \beta_{2} \mathrm{AR}, n=8 ; \mathrm{HCM} / \beta_{2} \mathrm{AR}$, $n=5$ ). (b) $\mathrm{HCM} \times \beta A R K c t$ mice (NTG, $n=6$; HCM, $n=8 ; \beta$ ARKct, $n=8$; $\mathrm{HCM} / \beta$ ARKct, $n=8$ ). (c) HCM $\times$ PLB-null mice (NTG, $n=8$; HCM, $n=7$; PLB-null, $n$ $=7$; HCM/PLB-null, $n=8)$. ${ }^{A} P<0.01$ vs. NTG; ANOVA. ${ }^{\mathrm{B}} P<0.05$ vs. HCM; ANOVA. pression ( $\sim 5$-fold) (22), and overexpression of $\mathrm{G}_{\alpha s}$, the signaling protein that couples $\beta$ ARs to adenylyl cyclase (23). Under physiological conditions, it is likely that the toxic effects of elevated adrenergic drive are mediated primarily by $\beta_{1}$ ARs, which have a higher affinity for norepinephrine and which primarily stimulate $G_{s}$, in contrast to $\beta_{2} \mathrm{AR}$ stimulation, which can strongly stimulate $G_{i}$ and may even be protective against cardiac myocyte apoptosis $(5,24)$. However, extremely high levels of $\beta_{2} \mathrm{AR}$ overexpression as in the TG4 mouse line used here ( $\sim 200$-fold) may cause aberrant receptor coupling that induces effects similar to the $\beta_{1} \mathrm{AR}$ signal. These results are consistent with recently reported findings that although chronically increased $\beta_{2}$ AR signaling might have therapeutic value in cardiomyopathy, receptor overexpression must be kept within a therapeutic window because of potential detrimental effects $(8,21)$. This is also consistent with recent reports showing that positive inotropes, when administered in low doses, can provide long-term therapeutic benefit (25).

\section{Figure 5}

Gene expression in cross-bred mouse hearts as assessed by slot blot of RNA extracted from left ventricular myocardium. All signals were normalized to GAPDH expression and are shown as fold of NTG. (a) $\mathrm{HCM} \times \beta_{2}$ AR mice (NTG, $\left.n=8 ; \mathrm{HCM}, n=10 ; \beta_{2} \mathrm{AR}, n=6 ; \mathrm{HCM} / \beta_{2} \mathrm{AR}, n=4\right)$. (b) $\mathrm{HCM} \times$ $\beta$ ARKct mice (NTG, $n=9$; HCM, $n=10 ; \beta$ ARKct, $n=10$; HCM $/ \beta$ ARKct, $n=$ 11). (c) HCM $\times$ PLB-null mice (NTG, $n=10$; HCM, $n=9$; PLB-null, $n=10$; HCM/PLB-null, $n=10)$. ${ }^{A} P<0.05$ vs. NTG; ANOVA. ${ }^{B} P<0.01$ vs. NTG; ANOVA. ${ }^{C} P<0.05$ vs. HCM; ANOVA.
In contrast to the toxic effects noted with high-level $\beta_{2} \mathrm{AR}$ overexpression, $\beta$ ARK inhibition and phospholamban ablation both prevented systolic dysfunction and exercise intolerance in the HCM mice. It is interesting to note that while PLB ablation completely prevented both cardiac dysfunction and histopathology in the MLP-null cardiomyopathic mice, $\beta$ ARK inhibition improved functional but not histological phenotypes $(6,7)$. In our experiments, functional improvements were noted in both HCM/PLB-null mice and HCM/BARKct mice despite the continued presence of histopathology typical of the parental HCM line. $\beta_{2} \mathrm{AR}$ overexpression, $\beta$ ARK inhibition, and phospholamban ablation are similar in that all three manipulations result in increased cardiac contraction and relaxation rates (13-15). One interpretation of the differing effects of these manipulations on disease progression in the HCM mice is that there is a therapeutic window for increased cardiac contraction or relaxation, i.e., that lesser amounts of contractile support are better tolerated than higher amounts. High-level $\beta_{2} A R$

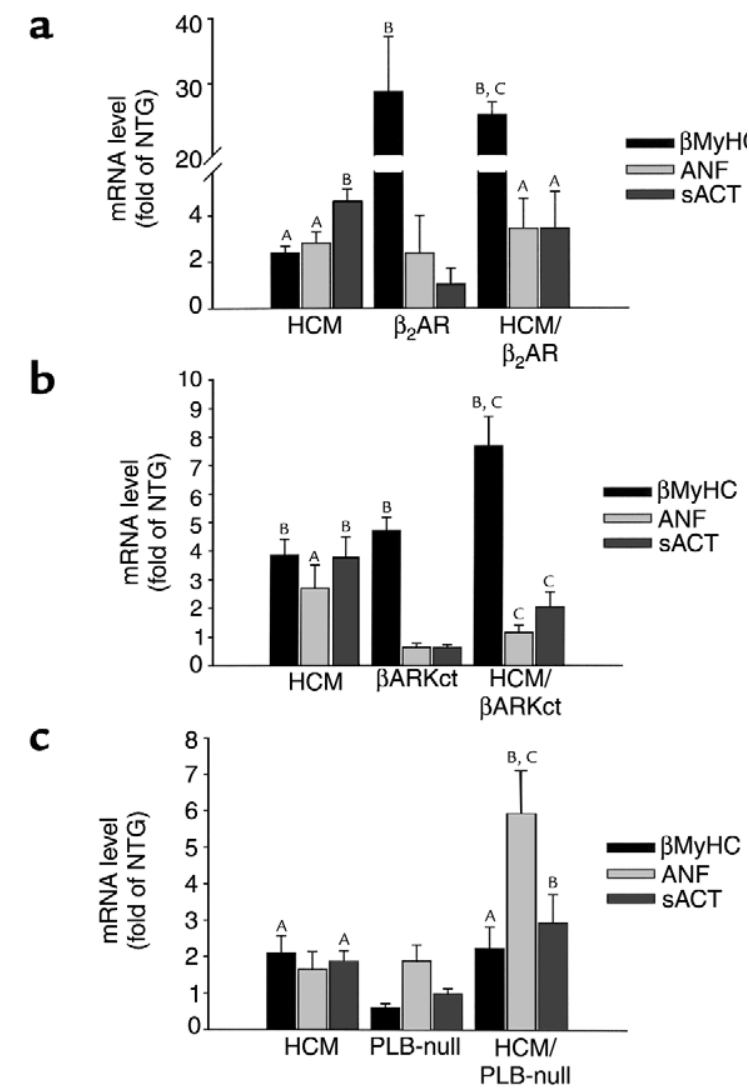


overexpression induced maximal cardiac contractile parameters, such that addition of isoproterenol did not further increase cardiac contraction or relaxation as measured in vivo by catheterization (13). In the HCM mice, $\beta_{2}$ AR overexpression initially increased inotropy to a greater extent than expression of $\beta A R K c t$ or PLB ablation, as the $\% \mathrm{FS}$ in 4-month-old $\mathrm{HCM} / \beta_{2} \mathrm{AR}$ mice was significantly higher than in NTG controls. Unlike $\beta_{2} A R$ overexpression, expression of $\beta$ ARKct caused a submaximal increase in both inotropic and lusitropic parameters, as isoproterenol administration caused further increases in both contraction and relaxation rates in vivo (14). Thus, despite increased basal $\beta$-adrenergic stimulation, $\beta$ ARKct animals retain some degree of $\beta$ AR-mediated inotropic reserve. PLB ablation was also associated with significantly enhanced basal contractile parameters and attenuated, but not abolished, responses to isoproterenol in vivo $(25,26)$. The \% FS in HCM/BARKct or HCM/PLBnull mice was not increased over that of NTG controls at any time point, although systolic function was significantly greater than in HCM littermates at 12 months of age. Maintenance of the ability of the $\beta A R$ signal system to regulate cardiac $\mathrm{Ca}^{2+}$ fluxes and contractile parameters in response to differing environmental conditions may be an important consideration in treating heart failure. Another possible explanation for the different outcomes of the three types of HCM crosses is that the site of the genetic/biologic inotropic or lusitropic effect is critical. This is potentially relevant to the $\mathrm{HCM} / \beta_{2} \mathrm{AR}$ versus the HCM/PLB-null animals, where in the case of the latter no increase in $\mathrm{Ca}^{2+}$ channel-mediated $\mathrm{Ca}^{2+}$ influx occurs, and diastolic $\mathrm{Ca}^{2+}$ levels would be expected to be decreased. This might lead to less of a tendency to activate $\mathrm{Ca}^{2+}$-dependent signaling pathways that culminate in cardiomyopathic alterations in gene expression (27). The lack of a chronotropic effect of PLB ablation (15) compared with $\beta_{2} \mathrm{AR}$ overexpression (13) also fits into this scenario, inasmuch as an increase in heart rate may be energetically unfavorable and/or arrhythmogenic.

It is also possible that the beneficial effects of PLB ablation or $\beta A R K$ inhibition via $\beta$ ARKct expression are due to effects in cardiac myocytes other than increased cardiac contractility. Clinical experience has shown that the direct stimulation of cardiac contractility (used by positive inotropic agents such as dobutamine and milrinone) does not provide long-term benefit, and the benefit observed via ACE inhibitors or $\beta$ AR-blockers is attributed to biologic effects, particularly the reversal of myocardial remodeling, rather than direct inotropic increases (4). Both ACE inhibitors and $\beta$ AR-blockers attenuate cardiac remodeling, and, in the case of some $\beta A R$ blockers, "reverse remodeling" has been reported, with regression of myocardial mass and normalization of ventricular shape (4). High-level $\beta_{2} A R$ overexpression clearly increased pathological remodeling in the HCM hearts, causing significant hypertrophy and dilation (Figure 1). In contrast, HCM $/ \beta$ ARKct mice were not hypertrophied to the same extent as HCM littermates were (Table 1). $\beta$ ARK inhibition also decreased the expression of two molecular markers of hypertrophy, ANF and $\alpha$-skeletal actin, in the HCM background (Figure 5). This suggests that $\beta$ ARK inhibition reversed hypertrophic remodeling in the HCM hearts.

In contrast, the improved functional phenotype of the HCM/PLB-null animals was accompanied by significant cardiac hypertrophy, with greater heart/body weight ratios than either HCM or PLB-null littermates. Given that both the HCM and PLB-null animals had significantly increased heart/body weights $(\sim 10 \%)$ compared with NTG littermate controls, the approximately $30 \%$ increase in heart/body weights in HCM/PLB-null littermates appears to be due to an additive effect, and it is interesting to speculate that the type of hypertrophy contributed by the HCM transgene is different from that contributed by PLB ablation. In the case of PLB-null mice, hypertrophy is not accompanied by increased expression of hypertrophic markers and histopathology, supporting the notion that the cardiac hypertrophy in PLB-null mice is physiologically different from that of the HCM mice. Further study of the differential nature of pathological hypertrophy in disease such as HCM versus nonpathological cardiac hypertrophy, such as that which may occur in PLB-null animals or in response to exercise training, is warranted.

Finally, our results strongly support the idea that $\beta A R K 1$ activity plays a key role in the progression of cardiac decompensation. Expression of the $\beta A R K c t$ peptide has now been shown to provide beneficial effects in several models of cardiomyopathy $(7,9)$. The $\beta A R K c t$ peptide contains the terminal 194 amino acids of $\beta$ ARK1, including a domain that binds and sequester $\mathrm{G}_{\beta \gamma}$, thereby preventing targeting of endogenous $\beta A R K 1$ to the sarcolemmal membrane. The $\mathrm{G}_{\beta \gamma}$-binding domain bears significant homology to the pleckstrin homology domains that are found in a variety of signaling molecules such as Ras-GAP, so it is possible that expression of the $\beta A R K c t$ peptide interferes with other protein-protein interactions in the cardiac myocyte (28). Although other effects of $\beta$ ARKct expression cannot be excluded, several lines of evidence suggest that inhibition of $\beta A R K 1$ is the primary mechanism for its salutary effects. $\beta$ ARK1 levels are increased in cardiac hypertrophy and heart failure (29), and we have previously reported increased $\beta A R K 1$ levels and activity in the hearts of 8-month-old male HCM mice (12). Besides the demonstrated ability of $\beta$ ARK1 to uncouple $\beta$ ARs and decrease cardiac contractility, $\beta$ ARK1 has also been shown to induce MAP kinase signaling in model systems (30). Members of the MAP kinase family have been implicated in cardiac muscle hypertrophy and in apoptosis (31), and we might speculate that the shunting of $\beta A R s$ to MAP kinase signal pathways may be responsible for triggering cardiac remodeling. In $\mathrm{HCM} / \beta_{2} \mathrm{AR}$ mice, the high level of $\beta_{2} \mathrm{AR}$ overexpression might increase the substrate for $\beta A R K 1$, which is elevated in the HCM hearts (12), leading to increased formation of $\beta A R / \beta a r r e s t i n / c-S r c$ complexes and subsequent MAP kinase signaling (30), triggering the rapidly progressive remodeling and heart failure we observed in 
these animals. Inhibition of $\beta$ ARK1 prevented cardiac hypertrophy in the HCM mice, consistent with this hypothesis. Interestingly, carvedilol, a third-generation $\beta$ AR-blocker that reverses remodeling and decreases longterm morbidity and mortality in heart failure (1), decreased cardiac $\beta$ ARK1 activity when administered to mice (32). Furthermore, in the calsequestrin overexpression model of heart failure, both $\beta$ ARK inhibition and $\beta$ adrenergic blockade provided salutary effects which were completely synergistic (33). $\beta$ ARK inhibition, but not $\beta_{2} \mathrm{AR}$ overexpression or phospholamban ablation, has also been shown to provide protective effects against ischemic injury $(34,35)$.

In conclusion, it seems likely that neither chronically enhanced $\beta A R$ signaling nor abnormally desensitized $\beta A R s$ will provide optimal cardiac function in the context of cardiomyopathy. Prevention of cardiac dysfunction in HCM mice by phospholamban ablation supports the idea that the phospholamban-SR $\mathrm{Ca}^{2+}$ ATPase interaction is a good target for therapy of heart disease. However, PLB is reduced in both the $\beta_{2} \mathrm{AR}$ overexpressers (36) and the $\beta A R K$ inhibitor mice (E.G. Kranias, unpublished observations), which have very different phenotypes, suggesting that decreasing PLB alone is not the sole basis for the phenotypes of the cross-bred mice described here. $\beta A R K 1$ inhibition not only prevented cardiac dysfunction, but it also prevented cardiac remodeling and hypertrophic gene expression in HCM mice. The finding of dramatic effects on the HCM cardiomyopathic phenotype by inhibition of a single molecule, $\beta$ ARK1, suggests that this protein may represent a therapeutic target with significant potential for preventing the progression of heart failure.

\section{Acknowledgments}

This work was supported in part by grants from the NIH (HL50560 to L.A. Leinwand; P40 RR12358 to E.G. Kranias, and HL61690 to W.J. Koch). R.J. Lefkowitz is an Investigator of the Howard Hughes Medical Institute.

1. Packer, M., et al. 1996. The effect of carvedilol on morbidity and mortality in patients with chronic heart failure. U.S. Carvedilol Heart Failure Study Group. N. Engl. J. Med. 334:1349-1355.

2. 1999. The Cardiac Insufficiency Bisoprolol Study II (CIBIS-II): a randomised trial. Lancet. 353:9-13.

3. 1999. Effect of metoprolol CR/XL in chronic heart failure: Metoprolol $\mathrm{CR} / \mathrm{XL}$ Randomised Intervention Trial in Congestive Heart Failure (MERIT-HF). Lancet. 353:2001-2007.

4. Bristow, M.R. 2000. beta-adrenergic receptor blockade in chronic heart failure. Circulation. 101:558-569.

5. Lefkowitz, R.J., Rockman, H.A., and Koch, W.J. 2000. Catecholamines, cardiac beta-adrenergic receptors, and heart failure. Circulation. 101:1634-1637.

6. Minamisawa, S., et al. 1999. Chronic phospholamban-sarcoplasmic reticulum calcium ATPase interaction is the critical calcium cycling defect in dilated cardiomyopathy. Cell. 99:313-322.

7. Rockman, H.A., et al. 1998. Expression of a beta-adrenergic receptor kinase 1 inhibitor prevents the development of myocardial failure in gene-targeted mice. Proc. Natl. Acad. Sci. USA. 95:7000-7005.

8. Dorn, G.W., Tepe, N.M., Lorenz, J.N., Koch, W.J., and Liggett, S.B. 1999. Low- and high-level transgenic expression of beta2-adrenergic receptors differentially affect cardiac hypertrophy and function in Galphaq-overexpressing mice. Proc. Natl. Acad. Sci. USA. 96:6400-6405.

9. Harding, V.B., Rapacciuolo, A., Mao, L., Lefkowitz, R.J., and Rockman, H. 1999. betaARK1 inhibition improves survival and cardiac function in a mouse model of severe cardiomyopathy. Circulation. 100(Suppl. I):I-
552. (Abstr.)

10. Solaro, R.J. 1999. Is calcium the 'cure' for dilated cardiomyopathy? Nat. Med. 5:1353-1354.

11. Vikstrom, K.L., Factor, S.M., and Leinwand, L.A. 1996. Mice expressing mutant myosin heavy chains are a model for familial hypertrophic cardiomyopathy. Mol. Med. 2:556-567.

12. Freeman, K., et al. 2001. Progression from hypertrophic to dilated cardiomyopathy in mice that express a mutant myosin transgene. Am. J. Physiol. 280:4151-4159.

13. Milano, C.A., et al. 1994. Enhanced myocardial function in transgenic mice overexpressing the beta 2-adrenergic receptor. Science. 264:582-586.

14. Koch, W.J., et al. 1995. Cardiac function in mice overexpressing the betaadrenergic receptor kinase or a beta ARK inhibitor. Science. 268:1350-1353.

15. Luo, W., et al. 1994. Targeted ablation of the phospholamban gene is associated with markedly enhanced myocardial contractility and loss of beta-agonist stimulation. Circ. Res. 75:401-409.

16. Tardiff, J.C., et al. 1999. Cardiac troponin T mutations result in allelespecific phenotypes in a mouse model for hypertrophic cardiomyopathy. J. Clin. Invest. 104:469-481.

17. Jones, W.K., et al. 1996. Ablation of the murine alpha myosin heavy chain gene leads to dosage effects and functional deficits in the heart. J. Clin. Invest. 98:1906-1917.

18. Desai, K.H., Schauble, E., Luo, W., Kranias, E., and Bernstein, D. 1999. Phospholamban deficiency does not compromise exercise capacity. Am. J. Physiol. 276:H1172-H1177.

19. Schwartz, K., et al. 1986. Alpha-skeletal muscle actin mRNA's accumulate in hypertrophied adult rat hearts. Circ. Res. 59:551-555.

20. Calderone, A., Takahashi, N., Izzo, N.J., Jr., Thaik, C.M., and Colucci, W.S. 1995. Pressure- and volume-induced left ventricular hypertrophies are associated with distinct myocyte phenotypes and differential induction of peptide growth factor mRNAs. Circulation. 92:2385-2390.

21. Liggett, S.B., et al. 2000. Early and delayed consequences of beta(2)adrenergic receptor overexpression in mouse hearts: critical role for expression level. Circulation. 101:1707-1714.

22. Engelhardt, S., Hein, L., Wiesmann, F., and Lohse, M.J. 1999. Progressive hypertrophy and heart failure in beta1-adrenergic receptor transgenic mice. Proc. Natl. Acad. Sci. USA. 96:7059-7064.

23. Iwase, M., et al. 1997. Cardiomyopathy induced by cardiac Gs alpha overexpression. Am. J. Physiol. 272:H585-H589.

24. Communal, C., Singh, K., Sawyer, D.B., and Colucci, W.S. 1999. Opposing effects of beta(1)- and beta(2)-adrenergic receptors on cardiac myocyte apoptosis: role of a pertussis toxin-sensitive $\mathrm{G}$ protein. Circulation. 100:2210-2212.

25. Lowes, B.D., et al. 2000. Low-dose enoximone improves exercise capacity in chronic heart failure. Enoximone Study Group. J. Am. Coll. Cardiol. 36:501-508.

26. Hoit, B.D., Khoury, S.F., Kranias, E.G., Ball, N., and Walsh, R.A. 1995. In vivo echocardiographic detection of enhanced left ventricular function in gene-targeted mice with phospholamban deficiency. Circ. Res. 77:632-637.

27. Lorenz, J.N., and Kranias, E.G. 1997. Regulatory effects of phospholamban on cardiac function in intact mice. Am. J. Physiol. 273:H2826-H2831.

28. Frey, N., McKinsey, T.A., and Olson, E.N. 2000. Decoding calcium signals involved in cardiac growth and function. Nat. Med. 6:1221-1227.

29. Inglese, J., Freedman, N.J., Koch, W.J., and Lefkowitz, R.J. 1993. Structure and mechanism of the $\mathrm{G}$ protein-coupled receptor kinases. J. Biol. Chem. 268:23735-23738.

30. Ungerer, M., et al. 1994. Expression of beta-arrestins and beta-adrenergic receptor kinases in the failing human heart. Circ. Res. 74:206-213.

31. Luttrell, L.M., et al. 1999. Beta-arrestin-dependent formation of beta2 adrenergic receptor-Src protein kinase complexes. Science. 283:655-661.

32. Wang, Y., et al. 1998. Cardiac muscle cell hypertrophy and apoptosis induced by distinct members of the p38 mitogen-activated protein kinase family. J. Biol. Chem. 273:2161-2168.

33. Harding, V., Jones, L.R., Lefkowitz, R.J., Koch, W.J., and Rockman, H.A 2001. Cardiac BARK inhibition improves survival and augments beta blocker therapy in a mouse model of severe heart failure. Proc. Natl. Acad. Sci. USA. In press.

34. Iaccarino, G., Tomhave, E.D., Lefkowitz, R.J., and Koch, W.J. 1998. Reciprocal in vivo regulation of myocardial $G$ protein-coupled receptor kinase expression by beta-adrenergic receptor stimulation and blockade. Circulation. 98:1783-1789.

35. Cross, H.R., Steenbergen, C., Lefkowitz, R.J., Koch, W.J., and Murphy, E. 1999. Overexpression of the cardiac beta(2)-adrenergic receptor and expression of a beta-adrenergic receptor kinase-1 (betaARK1) inhibitor both increase myocardial contractility but have differential effects on susceptibility to ischemic injury. Circ. Res. 85:1077-1084.

36. Cross, H.R., Steenbergen, C., Jr., and Murphy, E. 1999. Phospholamban knock-out (PLB-KO) mice exhibit increased susceptibility to myocardial ischemic injury: a gender-specific effect. Circulation. 100(Suppl. I):I-767. (Abstr.) 